\title{
Long-Term Clinical Outcome in Familial and Sporadic Papillary Thyroid Carcinoma
}

\author{
Marco Capezzone ${ }^{a} \quad$ Noemi Fralassi $^{a} \quad$ Chiara Secchi $^{a} \quad$ Silvia Cantara ${ }^{a}$ \\ Lucia Brilli $^{a}$ Tania Pilli ${ }^{a}$ Fabio Maino ${ }^{a}$ Raffaella Forleo ${ }^{a}$ Furio Pacini $^{a}$ \\ Gabriele Cevenini $^{b}$ Alessandra Cartocci ${ }^{b}$ Maria Grazia Castagna ${ }^{a}$ \\ ${ }^{a}$ Department of Medical, Surgical and Neurological Sciences, University of Siena, Siena, Italy; ${ }^{b}$ Department of \\ Medical Biotechnologies, University of Siena, Siena, Italy
}

\section{Keywords}

Familial papillary thyroid cancer - Sporadic papillary thyroid cancer · Outcome · Prognosis · Recurrences

\begin{abstract}
Background: The definition and the behaviour of familial papillary thyroid cancer (FPTC) compared to the sporadic form (SPTC) are still debated. Some authors believe that only families with 3 or more affected members represent an actual example of familial diseases. Objectives: The objective of the study was to analyse the clinicopathological features and the outcome of sporadic and familial PTC patients also according to the number of affected members. Methods: Among 731 patients, we identified 101 (13.8\%) with familial diseases, 79 with 2 affected members (FPTC-2) and 22 with 3 or more affected members (FPTC-3) followed for a mean period of 10 years. Results: FPTC patients had more frequently bilateral tumour ( $p=0.007$ ). No difference was found between the 2 groups for the other evaluated variables. At the time of the first follow-up (1-2 years after initial therapy), FPTC patients had a higher rate of persistent disease. However, at the last follow-up, the clinical outcome was not different between sporadic and familial patients. When the
\end{abstract}

comparison between SPTC and FPTC was performed, according to the number of affected members, a significant trend between the 3 groups was observed for tumour diameter $(p=0.002)$ and bilaterality $(p=0.003)$, while we did not observe a significant trend for both response to initial therapy $(p=0.15)$ and last clinical outcome $(p=0.22)$. Conclusions: Our results suggest that, although the clinicopathological features of FPTC may be more aggressive, the longterm outcome is similar between FPTC and SPTC. A possible explanation is that PTC has a favourable prognosis, even when clinical presentation is more aggressive.

(c) 2020 European Thyroid Association Published by S. Karger AG, Basel

\section{Introduction}

The incidence of non-medullary thyroid cancer, in particular papillary thyroid cancer (PTC), has increased in many countries over the past 20 years [1-3]. As a consequence, also the reported incidence of the familial form of PTC (FPTC), defined by the presence of the tumour in 2 or more first-degree relatives in absence of known hereditary syndromes associated with PTC, is more com- 


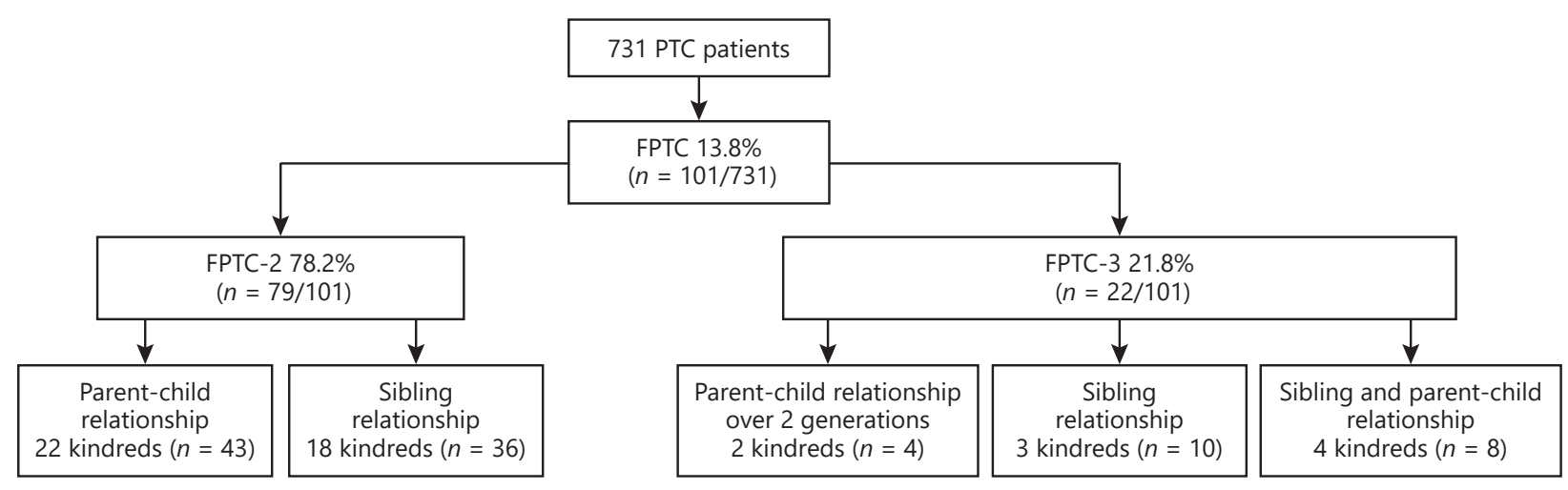

Fig. 1. Family relationship between familial papillary thyroid cancer (FPTC) patients.

mon nowadays than previously, ranging from 6 to $10 \%$ of all cases of PTC [4-6].

Although some authors did not find any difference between sporadic and familial PTC [7-9], the majority of the studies in the literature reported an increased aggressiveness of FPTC, compared with the sporadic form (SPTC), being usually characterized by a higher grade of multifocality, lymph node metastases and risk of recurrence during follow-up [10-14]. In our previous study [11], we reported a significant difference between SPTC and FPTC patients in terms of more frequent tumour multifocality and a worse clinical outcome in the shortterm follow-up in FPTC patients. Specifically, at a mean follow-up of 4.7 years, the rate of cured patients was $87.7 \%$ in sporadic and $67.6 \%$ in familial PTC.

The first aim of the present study was to evaluate and to compare the long-term clinical outcome of the same series of patients with FPTC in comparison with patients affected by SPTC. Moreover, the current definition of FPTC is still debated since it has been suggested that families with only 2 affected members may represent a coincidental association of the disease. Charkes et al. [15], using a mathematical simulation, reported that $62-69 \%$ of 2 -hit families are sporadic occurrences and, thus, only families with more than 3 affected first-degree relatives should be considered for clinical and genetic investigations of FPTC. On the contrary, other authors observed that PTC is a rare disease affecting between 1 in 10,000 and 1 in 20,000 subjects; thus, the probability that $2 \mathrm{mem}$ bers of a family would have a PTC (FPTC) by chance is between 1/10,000,000 and 1/40,000,000 [16]. Unfortunately, up to date, we have no genetic information able to discriminate between the familial and sporadic form of PTC. Therefore, the only peculiarity of FPTC consists in an increased aggressiveness compared to the sporadic form.

Assuming that FPTC with only 2 affected members represents a coincidental association of the disease, we hypothesized that the clinical presentation and outcome should be similar between SPTC and FPTC- 2 and a worse presentation and outcome should be observed only in the group of FPTC patients with 3 or more family members affected. To verify our hypothesis, the clinical presentation and outcome of SPTC patients were compared to FPTC with 2 affected members (FPTC-2) and to FPTC with 3 or more affected members (FPTC-3).

\section{Patients and Methods}

\section{Study Population}

We retrospectively reviewed the clinical records of 731 PTC patients followed at the Section of Endocrinology, University of Siena, Italy, from 1978 to 2018.

This cohort included 541/731 (74\%) females and 190/731 (26\%) males (F/M: 3/1), with age and mean follow-up ranging from 7 to 82 years (mean \pm SD: $46.6 \pm 15.6$ years) and from 2 to 28 years (mean \pm SD: $10.0 \pm 5.1$ years), respectively. We identified 101 FPTC (13.8\%) and 630 SPTC (86.2\%) patients. FPTC patients, belonging to 49 pedigrees, were defined by the presence of 2 or more first-degree relatives with thyroid cancer of follicular cell origin, after excluding clinical or pathological evidence of hereditary syndromes associated with PTC, such as familial adenomatous polyposis, Gardner syndrome, Peutz-Jeghers syndrome and Cowden disease. Patients with FPTC were subsequently divided into 2 subgroups according to the number of affected members: 79 (78.2\%) had 2 affected members in their family (FPTC-2) and the remain- 
ing $22(21.8 \%)$ had 3 or more affected family members in their family (FPTC-3). In the group of kindreds with only 2 affected members, a parent-child relationship was found in 22 families (43/44 family members with available data) and a sibling relationship was found in 18 kindreds ( 36 members). In the pedigrees with 3 or more affected family members, we found a sibling relationship in 3 kindreds (10/11 members), a parent-child relationship over 2 generations in 2 kindreds (4/6 members with available data) and 4 kindreds with both sibling and parent-child relationship (8/12 members with available data) (Fig. 1).

A written consent was given by all patients to use their clinical data for research purpose.

\section{Initial Therapy and Follow-Up}

Initial treatment was represented by near total thyroidectomy (with or without lymph node dissection) for 719/731 (98.3\%) patients followed by ${ }^{131}$ I remnant ablation in 591/731 (80.8\%) of cases. Patients who underwent radioactive iodine therapy received a therapeutic dose of ${ }^{131}$ I after levothyroxine withdrawal in 224/591 (37.9\%) cases and after recombinant human TSH (rhTSH) administration in $367 / 591$ (62.1\%) cases. Each patient was risk stratified according to the American Thyroid Association (ATA) risk stratification system [17]. Three hundred seventy-nine/731 (51.8\%) patients were classified at low risk, 350/731 (47.9\%) patients at intermediate risk and 2/731 (0.3\%) patients at high risk. Patients were followed every 6 months during the first year; subsequently, the frequency of the follow-up visits was based on the clinical course of the disease and the estimated risk of recurrence for each individual patient.

Clinical outcomes were assessed at 2 time intervals: after initial therapy and at the last follow-up. The response to the initial therapy based on clinical data obtained in the first 2 years of follow-up and clinical status at the last follow-up were assessed using a restratification system proposed by the 2016 ATA guidelines [18]. Specifically, patients were classified as having, respectively, an excellent response if there was no clinical, biochemical or structural evidence of disease; a biochemical incomplete response if there were abnormal thyroglobulin or rising anti-thyroglobulin antibody levels in the absence of localizable disease; a structural incomplete response if there were persistent or newly identified locoregional or distant metastases and, finally, an indeterminate response if there were non-specific biochemical or structural findings that could not be confidently classified as either benign or malignant. This included patients with stable or declining anti-thyroglobulin antibody levels without definitive structural evidence of disease. For statistical analysis, the last 3 categories were grouped.

\section{Statistical Analysis}

Epidemiological quantitative data are presented as the mean \pm SD and median. The following variables were studied by univariate analysis: age at diagnosis, gender, tumour diameter, tumour extension, multifocality, bilaterality, lymph node dissection and lymph node metastases and 2009 ATA risk, radioiodine ablation, doses of ablative ${ }^{131} \mathrm{I}$, cumulative activity of ${ }^{131} \mathrm{I}$ and the length of follow-up. To evaluate the difference between SPTC/FPTC, the Mann-Whitney U test was performed; instead, for the difference between SPTC/FPTC-2/ FPTC-3, the Kruskal-Wallis test and the post hoc Dunn's test were used. To evaluate significant differences in data frequency, we analysed contingency tables. Tables with size $2 \times 2$ were examined by the $\chi^{2}$ test or a numerical approximation of the Fisher exact test. To evaluate the association with SPTC/FPTC-2/FPTC-3 variables (table
Table 1. Demographic and clinicopathological features of SPTC and FPTC patients

\begin{tabular}{|c|c|c|c|}
\hline Parameters & $\begin{array}{l}\text { SPTC } \\
(n=630)\end{array}$ & $\begin{array}{l}\text { FPTC } \\
(n=101)\end{array}$ & $p$ \\
\hline Gender, $n(\%)$ & & & 0.9 \\
\hline Female & $466(74)$ & $75(74.3)$ & \\
\hline Age at diagnosis, years & & & 0.2 \\
\hline Mean \pm SD & $46.6 \pm 15.6$ & $44.4 \pm 15.7$ & \\
\hline Range & $7-82$ & $14-81$ & \\
\hline Median & 46 & 44 & \\
\hline Tumour diameter, $\mathrm{cm}$ & & & 0.6 \\
\hline Mean \pm SD & $1.6 \pm 1.2$ & $1.5 \pm 1.1$ & \\
\hline Range & $0.1-7.2$ & $0.1-6.5$ & \\
\hline Median & 1.3 & 1.1 & \\
\hline Multifocality, $n(\%)$ & & & 0.09 \\
\hline Yes & $238(37.8)$ & $47(46.5)$ & \\
\hline Bilaterality, $n(\%)$ & & & 0.007 \\
\hline Yes & $168(26.7)$ & $40(39.6)$ & \\
\hline Tumour extension, $n(\%)$ & & & 0.4 \\
\hline Extrathyroidal & $227(36.1)$ & $40(39.6)$ & \\
\hline \multicolumn{4}{|c|}{ Lymph node dissection, $n(\%)$} \\
\hline Therapeutic & $100(15.9)$ & $20(19.8)$ & 0.6 \\
\hline Prophylactic & $52(8.3)$ & $8(7.9)$ & \\
\hline Not performed & $478(75.8)$ & $73(72.3)$ & \\
\hline \multicolumn{4}{|c|}{ Lymph node metastases, $n(\%)$} \\
\hline Yes & $159(25.2)$ & $28(27.7)$ & 0.6 \\
\hline ATA risk, $n(\%)$ & & & 0.9 \\
\hline High & $2(0.3)$ & 0 & \\
\hline Intermediate & $301(47.8)$ & $49(48.5)$ & \\
\hline Low risk & $327(51.9)$ & $52(51.5)$ & \\
\hline \multicolumn{4}{|l|}{ Radioiodine ablation, $n(\%)$} \\
\hline Yes & $507(80.5)$ & $84(83.2)$ & 0.5 \\
\hline \multicolumn{4}{|c|}{ Doses of ablative ${ }^{131} \mathrm{I}(\mathrm{mCi}), n(\%)$} \\
\hline Mean \pm SD & $70.5 \pm 33.9$ & $73.6 \pm 46.4$ & 0.6 \\
\hline Range & $15-300$ & $15-300$ & \\
\hline Median & 65 & 65 & \\
\hline \multicolumn{4}{|c|}{ Cumulative activity of ${ }^{131} \mathrm{I}(\mathrm{mCi}), n(\%)$} \\
\hline Mean \pm SD & $129.9 \pm 186.0$ & $158.2 \pm 179.9$ & 0.09 \\
\hline Range & $15-1300$ & $15-1056$ & \\
\hline Median & 80 & 100 & \\
\hline Follow-up, years & & & 0.1 \\
\hline Mean \pm SD & $10.1 \pm 5.1$ & $9.3 \pm 4.9$ & \\
\hline Range & $2-28$ & $2-25$ & \\
\hline Median & 10 & 9 & \\
\hline
\end{tabular}

with size $2 \times 3$ ), the $\chi^{2}$ test for trend was performed. Using the G*Power software, we evaluated the minimum sample size. Predetermined power of $80 \%$ and a significance level of $95 \%$ were set, and we found a number of at least 700 patients for an effect size close to 0.1 . With our cohort of 731 patients, an effect size of 0.104 was found.

Statistical analysis was performed using the software StatView for Windows version 5.0.1 (SAS Institute, Cary, NC, USA) and the SPSS Statistics version 22.0. A $p$ value $<0.05$ was considered statistically significant. 
Table 2. Clinical outcomes of SPTC and FPTC patients

\begin{tabular}{|c|c|c|c|}
\hline Response to initial therapy, $n(\%)$ & $n=630$ & $n=86$ & \multirow[t]{3}{*}{0.044} \\
\hline Excellent response & $450(71.4 \%)$ & $52(60.5 \%)$ & \\
\hline Indeterminate/biochemical incomplete response/structural incomplete response & $180(28.6 \%)$ & $34(39.5 \%)$ & \\
\hline Clinical outcomes at the end of follow-up, $n(\%)$ & $n=626$ & $n=82$ & \multirow[t]{2}{*}{0.26} \\
\hline Excellent response & $528(84.3 \%)$ & $65(79.3 \%)$ & \\
\hline
\end{tabular}

\section{Results}

\section{Comparison between FPTC and SPTC Patients}

The clinicopathological features of FPTC $(n=101)$ and SPTC patients $(n=630)$ are shown in Table 1. FPTC patients had more frequently bilateral tumour $(p=0.007)$. No difference was found between the 2 groups regarding gender, age at diagnosis, tumour diameter, multifocality, tumour extension, lymph node dissection and lymph node metastases, ATA risk, radioiodine ablation, doses of ablative ${ }^{131} \mathrm{I}$, cumulative activity of ${ }^{131} \mathrm{I}$ administered and the length of follow-up.

At the time of the first follow-up (1-2 years after initial therapy), the clinical status was significantly different between the 2 groups $(p=0.04)$. In particular, 52/86 (60.5\%) patients with FPTC and 450/630 (71.4\%) patients with SPTC fulfilled the criteria of excellent response. Indeterminate or biochemical or structural incomplete response were found in 34/86 (39.5\%) patients with FPTC and in $180 / 630(28.6 \%)$ patients with SPTC (Table 2). We performed the same analysis according to the initial risk class of PTC patients. We observed that in the low-risk group, the rate of patients with worse outcome at 2 years of follow-up was slightly higher in FPTC than SPTC patients ( 28.3 and $15.9 \%$, respectively, $p=0.05$ ), while no significant differences between FPTC and SPTC patients were observed in the subgroup of intermediate-risk patients (52.5 and $42.2 \%$, respectively, $p=0.23$ ).

Four patients were lost during follow-up in both groups (FPTC and SPC), and no patients in the entire study died from their PTC. At the last follow-up, the FPTC group included 82 patients and the SPTC group 626 patients.

At the last follow-up (median follow-up of 10 years), 65/82 (79.3\%) patients with FPTC and 528/626 (84.3\%) patients with SPTC had an excellent response. Indeterminate or biochemical or structural incomplete response was found in 17/82 (20.7\%) FPTC patients and in 98/626
(15.7\%) SPTC patients, without significant differences between SPTC and FPTC patients $(p=0.26)$. We performed the same analysis according to the initial risk class of PTC patients. We observed that in the low-risk group, the rate of patients with worse outcome at the last followup was higher in FPTC than in SPTC patients (13.3 and $7.4 \%$, respectively, $p=0.23$ ), while no significant differences between FPTC and SPTC patients were observed in the subgroup of intermediate-risk patients (29.7 and $24.5 \%$, respectively, $p=0.54$ ).

\section{Comparison between SPTC and FPTC according to} the Number of Affected Members

As shown in Table 3, the bilaterality rate showed a significant increasing trend $(p=0.03)$ from SPTC to FPTC3 . A significant trend between the 3 groups was also observed for tumour diameter $(p=0.002)$, in particular between FPTC-3 and SPTC or FPTC-2 (Table 3). On the contrary, no differences were found between the 3 groups regarding gender, age at diagnosis, multifocality, tumour extension, lymph node dissection and metastases, ATA risk class, radioiodine ablation, ablative activity of ${ }^{131} \mathrm{I}$, cumulative activity of ${ }^{131} \mathrm{I}$ administered and the length of follow-up.

We did not observe a significant trend in the response to initial therapy $(p=0.15)$ from SPTC to FPTC-3 (Table 4 ), probably due to the high rate of excellent response in FPTC-3 patients $(76.5 \%)$ that was similar to SPTC patients $(71.4 \%)$. Similarly, we did not find a significant trend in the outcomes at the end of follow-up between the 3 groups of patients $(p=0.22)$ (Table 4$)$.

\section{Discussion}

This study represents an extension of our previous report in which the clinical features at diagnosis and the short-term outcome of patients with sporadic and famil- 
Table 3. Comparison between demographic and clinicopathological features of SPTC, FPTC-2 and FPTC-3 patients

\begin{tabular}{|c|c|c|c|c|}
\hline Parameters & $\begin{array}{l}\text { SPTC } \\
(n=630)\end{array}$ & $\begin{array}{l}\text { FPTC-2 } \\
(n=79)\end{array}$ & $\begin{array}{l}\text { FPTC-3 } \\
(n=22)\end{array}$ & $p$ \\
\hline Gender, $n(\%)$ & & & & 0.99 \\
\hline Female & $466(74)$ & $59(74.7)$ & $6(27.3)$ & \\
\hline Age at diagnosis, years & & & & 0.42 \\
\hline Mean \pm SD & $46.6 \pm 15.6$ & $44.4 \pm 16.9$ & $44.5 \pm 10.2$ & \\
\hline Range & $7-82$ & $14-81$ & $23-70$ & \\
\hline Median & 46 & 43 & 45 & \\
\hline Tumour diameter, cm & & & & $0.02^{\mathrm{a}, \mathrm{b}}$ \\
\hline Mean \pm SD & $1.6 \pm 1.2$ & $1.6 \pm 1.2$ & $1.0 \pm 0.8$ & \\
\hline Range & $0.1-7.2$ & $0.1-6.5$ & $0.2-3$ & \\
\hline Median & 1.3 & 1.2 & 0.8 & \\
\hline Multifocality, $n(\%)$ & & & & 0.23 \\
\hline Yes & $238(37.8)$ & $39(49.4)$ & $8(36.4)$ & \\
\hline Bilaterality, $n(\%)$ & & & & 0.03 \\
\hline Yes & $168(26.7)$ & $33(41.8)$ & $7(31.8)$ & \\
\hline Tumour extension, $n(\%)$ & & & & 0.85 \\
\hline Extrathyroidal & $227(36.1)$ & $34(43.0)$ & $6(27.3)$ & \\
\hline Lymph node dissection, $n(\%)$ & & & & 0.84 \\
\hline Therapeutic & $100(15.9)$ & $16(20.2)$ & $4(18.2)$ & \\
\hline Prophylactic & $52(8.3)$ & $6(7.6)$ & $2(9.1)$ & \\
\hline Not Performed & $478(75.8)$ & $57(72.2)$ & $16(72.7)$ & \\
\hline Lymph node metastases, $n(\%)$ & & & & 0.63 \\
\hline Yes & $159(25.2)$ & $22(27.8)$ & $6(27.3)$ & \\
\hline ATA risk class, $n(\%)$ & & & & 0.71 \\
\hline High/intermediate & $303(48.1)$ & $41(51.9)$ & $8(36.4)$ & \\
\hline Low risk & $327(51.9)$ & $38(48.1)$ & $14(63.6)$ & \\
\hline Radioiodine ablation, $n(\%)$ & & & & 0.28 \\
\hline Yes & $504(80.5)$ & $73(92.4)$ & $16(72.7)$ & \\
\hline Doses of ablative ${ }^{131} \mathrm{I}(\mathrm{mCi}), n(\%)$ & & & & 0.13 \\
\hline Mean \pm SD & $70.5 \pm 33.9$ & $77.5 \pm 48.4$ & $54.6 \pm 29.6$ & \\
\hline Range & $15-300$ & $15-300$ & $30-100$ & \\
\hline Median & 65 & 75.5 & 50 & \\
\hline Cumulative activity of ${ }^{131} \mathrm{I}(\mathrm{mCi}), n(\%)$ & & & & 0.18 \\
\hline Mean \pm SD & $129.9 \pm 186.0$ & $157 \pm 189.4$ & $164.5 \pm 127.6$ & \\
\hline Range & $15-1300$ & 15-1056 & $30-460$ & \\
\hline Median & 80 & 100 & 150 & \\
\hline Follow-up, years & $10.3 \pm 5.6$ & $9.5 \pm 4.8$ & $8.1 \pm 4.8$ & 0.19 \\
\hline Range & $2-56$ & $2-25$ & $2-20$ & \\
\hline Median & 10 & 9 & 8 & \\
\hline
\end{tabular}

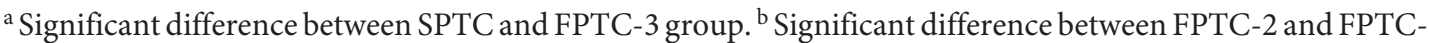
3 group.

ial PTC were compared. In our previous work, we demonstrated that FPTC patients showed more aggressive tumour phenotype, a worse outcome and presented features of clinical "anticipation" with the second generation developing the disease at an earlier age [11]. Recently, we also demonstrated that familial microPTC is character- ized by aggressive phenotype, in contrast to sporadic microPTC which has usually a more indolent behaviour [19]. Wang et al. [13] performed a recent meta-analysis that considered 12 studies with a total of 12,741 patients and reported that familial non-medullary thyroid cancer had a more aggressive biological presentation than SPTC, 
Table 4. Outcomes of patients with SPTC, FPTC-2 and FPTC3 at the time of first follow-up and at the end of follow-up

\begin{tabular}{|c|c|c|c|c|}
\hline Parameters & SPTC & FPTC-2 & FPTC-3 & $p$ \\
\hline Response to initial therapy, $n(\%)$ & $n=630$ & $n=69$ & $n=17$ & 0.15 \\
\hline Excellent response & $450(71.4 \%)$ & $39(56.5 \%)$ & $13(76.5 \%)$ & \\
\hline Indeterminate/biochemical incomplete response/structural incomplete response & $180(28.6 \%)$ & $30(43.5 \%)$ & $4(23.5 \%)$ & \\
\hline Clinical outcomes at the end of follow-up, $n(\%)$ & $n=626$ & $n=65$ & $n=17$ & 0.22 \\
\hline Excellent response & $528(84.3 \%)$ & $52(80.0 \%)$ & $13(76.5 \%)$ & \\
\hline Indeterminate/biochemical incomplete response/structural incomplete response & $98(15.7 \%)$ & $13(20.0 \%)$ & $4(23.5 \%)$ & \\
\hline
\end{tabular}

characterized by younger age at diagnosis, a higher incidence of multifocality, extrathyroidal invasion, bilateral disease and lymph node metastases at presentation. The results of the present study confirm that FPTC patients have more frequently bilateral tumour and worse outcome, at least in the short-term follow-up (2 years after surgical therapy and radioiodine ablation). Moreover, this cohort of patients had a longer follow-up, and this allowed us to have additional information and also to evaluate the clinical outcome after additional treatments in the subgroup of FPTC patients with persistent disease after initial therapy.

In our study, a worse outcome of FPTC patients was still observed up to a median follow-up of 5 years (data not shown). However, the clinical outcome of patients with FPTC was not significantly different from that observed in patients with SPTC at a median follow-up of 10 years. To our knowledge, only few studies compared the long-term clinical outcome between FPTC and SPTC patients and, similarly to our results, no differences were reported between the 2 groups of PTC patients [10,20].

In order to evaluate a possible impact of the small number of familial nonmedullary thyroid cancer (FNMTC) in our results, we performed a post hoc power analysis and we found that with our simple size, we were able to obtain a significant difference between sporadic and familial PTC only if the clinical outcome (i.e., excellent response) was at least $10 \%$ different between the 2 groups. This explains why we did not find any differences in terms of clinical outcome at the final follow-up, while this difference was observed at the time of the first evaluation after initial therapy.

On these bases, we can suppose that FPTC is more aggressive than SPTC as demonstrated by the worse clinical presentation and short-term outcome. However, if adequately treated (additional treatment during follow-up when necessary), the final outcome was not different from that observed in the sporadic form of PTC. Never- theless, also considering a low rate of persistent disease after initial treatment, we do not suggest a more aggressive treatment in all patients with FPTC, while we believe that a patient personalized approach might be more useful.

In the present study, we also compared the biological presentation and the clinical outcome between SPTC and FPTC patients with 2 affected first-degree relatives (FPTC-2) and 3 or more affected first-degree relatives (FPTC-3). We found no significant trend comparing outcome at initial follow-up and at the end of follow-up between the 3 groups. In particular, focusing on the response to initial therapy, the absence of a significant trend indicates that a rising number of affected family members probably is not a predictor of a worse clinical outcome. In our opinion, this observation strengthens the concept that even families with only 2 affected members should be considered as actual familial diseases and not a coincidental association of sporadic cases. In addition, the observation that, in the majority of the studies, familial patients belonging to kindreds with only 2 affected family members showed a more aggressive tumour phenotype in FPTC than in SPTC patients could be an additional demonstration that FPTC is a distinct entity compared to SPTC regardless of the number of members affected in the families $[8,12]$. Few studies have analysed the potential differences in the clinical presentation and outcome between families with only 2 affected members compared to families with 3 or more affected members, and in the majority of them, no differences were observed. Similarly, in our cohort of FPTC patients, no differences were found in the clinical behaviour of FPTC-2 and FPTC-3 patients. Moreover, it is important to highlight that our findings, as well as the results reported in previous studies, could be related to the limited number of subjects with more than 3 affected family members included [21-24] and also to the higher rate of microcarcinoma in the FPTC-3 group. Similarly to our results, a recent study which com- 
pared families with only 2 affected first-degree relatives $(n=93)$ with families with 3 or more affected first-degree relatives $(n=20)$ reported a higher proportion of papillary thyroid microcarcinomas in patients with more than 2 affected members [21]. This higher rate of microPTC documented in FPTC patients with 3 or more affected members is probably related to the screening effect.

Our study has some limitations that should be considered when interpreting our findings. Firstly, as already mentioned, the number of patients with 3 or more family members affected was small. This limitation has also been observed in previous studies, but given the rarity of this type of disease, only multicenter studies could allow a more reliable comparison by collecting several patients.

Secondly, some limitations are related to the retrospective nature of the study (e.g., loss of some patients during follow-up). On the other hand, our study has several strengths, including a similar initial therapeutic approach and follow-up strategy carried out at the same institution. PTC patients in our study were treated with near-total thyroidectomy followed in most cases by radioiodine remnant ablation, ruling out the bias of a different therapeutic approach between familial and sporadic PTC. Thirdly, it should be pointed out that, in our series, patients were submitted to total thyroidectomy followed by radioiodine remnant ablation in the majority of cases. In addition, FPTC patients tended, although not reaching statistical significance, to receive a greater cumulative activity of radioiodine. This more aggressive initial treatment in FPTC together with additional treatments when necessary could explain the good clinical outcome in the long-term follow-up, similar to that observed in SPTC patients. Additional studies including FPTC patients treated according to the current guideline recommendations (less total thyroidectomy and less radioiodine treatments at diagnosis) are necessary to clarify if the good outcome observed in our study is related to the more aggressive initial treatment of FPTC patients.
In conclusion, we confirm an increased aggressiveness of FPTC compared to the sporadic form, but the longterm outcome is similar in the 2 groups. The discovery of a familial predisposition to cancer supports the importance of taking family history as part of any routine assessment of the patient. A simple pedigree may alert the physician towards an increased familial risk. We suggest that more consideration should be given to the decision about adequate initial treatment for FPTC patients. A better understanding of the genetic basis of FPTC and further studies that compare the different types of FPTC kindreds could help reach a better risk stratification and guide surveillance and management plan.

\section{Statement of Ethics}

All patients have given their written informed consent, and the study protocol was approved by the institute's committee on human research (Comitato Etico Regione Toscana - Area Vasta Sud Est).

\section{Disclosure Statement}

The authors have no conflicts of interest to declare.

\section{Funding Sources}

This study did not receive any funding.

\section{Author Contributions}

M.C. and M.G.C. conceived of the presented idea and wrote the manuscript; N.F., C.S. and S.C. verified the analytical methods. L.B., T.P., F.M. and R.F. contributed to the design and implementation of the research; G.C. and A.C. performed the statistical analysis. All authors discussed the results and contributed to the final manuscript.

\section{References}

1 Davies L, Morris L, Hankey B. Increases in Thyroid Cancer Incidence and Mortality. JAMA. 2017 Jul;318(4):389-390.

2 Kitahara CM, Sosa JA. The changing incidence of thyroid cancer. Nat Rev Endocrinol. 2016 Nov; $12(11): 646-53$

3 Lim H, Devesa SS, Sosa JA, Check D, Kitahara CM. Trends in Thyroid Cancer Incidence and Mortality in the United States, 1974-2013. JAMA. 2017 Apr;317(13):1338-48.

Outcome of Familial Papillary Thyroid Cancer
4 Guilmette J, Nosé V. Hereditary and familial thyroid tumours. Histopathology. 2018 Jan; 72(1):70-81.

5 Klubo-Gwiezdzinska J, Yang L, Merkel R, Patel D, Nilubol N, Merino MJ, et al. Results of Screening in Familial Non-Medullary Thyroid Cancer. Thyroid. 2017 Aug;27(8):1017-24.

6 Peiling Yang S, Ngeow J. Familial non-medullary thyroid cancer: unraveling the genetic maze. Endocr Relat Cancer. 2016 Dec; 23(12):R577-95.
7 Pinto AE, Silva GL, Henrique R, Menezes FD, Teixeira MR, Leite V, et al. Familial vs sporadic papillary thyroid carcinoma: a matchedcase comparative study showing similar clini$\mathrm{cal} /$ prognostic behaviour. Eur J Endocrinol. 2013 Dec;170(2):321-7.

8 Moses W, Weng J, Kebebew E. Prevalence, clinicopathologic features, and somatic genetic mutation profile in familial versus sporadic nonmedullary thyroid cancer. Thyroid. 2011 Apr;21(4):367-71. 
9 Pitoia F, Cross G, Salvai ME, Abelleira E, Niepomniszcze H. Patients with familial nonmedullary thyroid cancer have an outcome similar to that of patients with sporadic papillary thyroid tumors. Arq Bras Endocrinol Metabol. 2011 Apr;55(3):219-23.

10 Uchino S, Noguchi S, Kawamoto H, Yamashita $\mathrm{H}$, Watanabe S, Yamashita H, et al. Familial nonmedullary thyroid carcinoma characterized by multifocality and a high recurrence rate in a large study population. World J Surg. 2002 Aug;26(8):897-902.

11 Capezzone M, Marchisotta S, Cantara S, Busonero G, Brilli L, Pazaitou-Panayiotou K, et al. Familial non-medullary thyroid carcinoma displays the features of clinical anticipation suggestive of a distinct biological entity. Endocr Relat Cancer. 2008 Dec;15(4): 1075-81.

12 McDonald TJ, Driedger AA, Garcia BM, Van Uum SH, Rachinsky I, Chevendra V, et al. Familial papillary thyroid carcinoma: a retrospective analysis. J Oncol. 2011;2011(Oct): 948786.

13 Wang X, Cheng W, Li J, Su A, Wei T, Liu F, et al. Endocrine tumours: familial nonmedullary thyroid carcinoma is a more aggressive disease: a systematic review and meta-analysis. Eur J Endocrinol. 2015 Jun;172(6):R25362.

14 Cao J, Chen C, Chen C, Wang QL, Ge MH Clinicopathological features and prognosis of familial papillary thyroid carcinoma-a large-scale, matched, case-control study. Clin Endocrinol (Oxf). 2016 Apr;84(4):598-606.
15 Charkes ND. On the prevalence of familial nonmedullary thyroid cancer in multiply affected kindreds. Thyroid. 2006 Feb;16(2): 181-6.

16 Moore FD Jr. Inherited aspects of papillary thyroid carcinoma. J Surg Oncol. 2006 Dec; 94(8):719-24

17 Cooper DS, Doherty GM, Haugen BR, Kloos RT, Lee SL, Mandel SJ, et al.; American Thyroid Association (ATA) Guidelines Taskforce on Thyroid Nodules and Differentiated Thyroid Cancer. Revised American Thyroid Association management guidelines for patients with thyroid nodules and differentiated thyroid cancer. Thyroid. 2009 Nov;19(11):1167214.

18 Haugen BR, Alexander EK, Bible KC, Doherty GM, Mandel SJ, Nikiforov YE, et al. 2015 American Thyroid Association Management Guidelines for Adult Patients with Thyroid Nodules and Differentiated Thyroid Cancer: The American Thyroid Association Guidelines Task Force on Thyroid Nodules and Differentiated Thyroid Cancer. Thyroid. 2016 Jan;26(1):1-133.

19 Capezzone M, Secchi C, Fralassi N, Cantara S, Brilli L, Ciuoli C, et al. Should familial disease be considered as a negative prognostic factor in micropapillary thyroid carcinoma? J Endocrinol Invest. 2019 Oct;42(10):1205-13.
20 Robenshtok E, Tzvetov G, Grozinsky-Glasberg S, Shraga-Slutzky I, Weinstein R, Lazar $\mathrm{L}$, et al. Clinical characteristics and outcome of familial nonmedullary thyroid cancer: a retrospective controlled study. Thyroid. 2011 Jan;21(1):43-8.

21 Lee YM, Yoon JH, Yi O, Sung TY, Chung KW, Kim WB, et al. Familial history of non-medullary thyroid cancer is an independent prognostic factor for tumor recurrence in younger patients with conventional papillary thyroid carcinoma. J Surg Oncol. 2014 Feb;109(2): 168-73.

22 Park YJ, Ahn HY, Choi HS, Kim KW, Park DJ, Cho BY. The long-term outcomes of the second generation of familial nonmedullary thyroid carcinoma are more aggressive than sporadic cases. Thyroid. 2012 Apr;22(4): 356-62.

23 Alsanea O, Wada N, Ain K, Wong M, Taylor $\mathrm{K}$, Ituarte $\mathrm{PH}$, et al. Is familial non-medullary thyroid carcinoma more aggressive than sporadic thyroid cancer? A multicenter series. Surgery. 2000 Dec;128(6):1043-50.

24 Mazeh H, Benavidez J, Poehls JL, Youngwirth L, Chen H, Sippel RS. In patients with thyroid cancer of follicular cell origin, a family history of nonmedullary thyroid cancer in one firstdegree relative is associated with more aggressive disease. Thyroid. 2012 Jan;22(1):3-8. 